\title{
Cervical Posture and Skeletal Malocclusions - Is there a Link?
}

\author{
Sanam Tauheed, ${ }^{1}$ Attiya Shaikh, ${ }^{2}$ Mubassar Fida ${ }^{3}$ \\ ${ }^{1}$ Head of Department, Jinnah Medical and Dental College, Karachi, Sindh, Pakistan, ${ }^{2}$ Head of Department, Liaquat \\ College of Medicine and Dentistry, Pakistan, ${ }^{3}$ Professor Orthodontics, Director Residency Program, The Aga Khan \\ University Hospital, Pakistan.
}

\begin{abstract}
Background: The present study was conducted in order to determine cervical posture in different skeletal sagittal malocclusions as well as to assess whether a correlation existed between cervical posture and skeletal relationships. Methods: Cervical curvature and inclination of 63 subjects was assessed using their lateral cephalometric radiographs. Cervical inclination was assessed using the cervicohorizontal postural variables namely OPT/HOR and CVT/HOR whereas cervical curvature was determined by measuring the angle OPT/ CVT. Sagittally, the subjects were also categorized into skeletal Class I, II and III based on the angle ANB. One way ANOVA was used for the comparison of cervical posture in different skeletal sagittal malocclusions. Pearson's correlation was used to evaluate the correlation of cervical posture with different skeletal sagittal jaw relations. Statistical significance level was set at $\mathrm{p} \leq 0.05$. Results: Statistically significant differences were found between the different skeletal malocclusions for the cervical curvature angle OPT/ CVT ( $\mathrm{p}=0.025)$. A weak correlation of cervical curvature angle OPT/CVT $(\mathrm{r}=0.305, \mathrm{p}=0.016)$ with sagittal malocclusion was found. Conclusions: Skeletal sagittal malocclusions differ in their cervical postures, especially cervical curvature. Skeletal Class III subjects have significantly straighter cervical columns than skeletal Class I subjects. Cervical curvature is correlated with sagittal jaw relations.
\end{abstract}

Keywords: cervical curvature; cervical posture; skeletal sagittal malocclusions .

\section{INTRODUCTION}

The alignment of the cervical vertebral column has been observed to have an inward curvature known as lordosis of the column with the convexity of the curve facing anteriorly. Most cervical lordosis involves the superior cervical vertebrae, $\mathrm{C} 1$ and $\mathrm{C} 2$, whereas only $15 \%$ of the lordosis occurs at the lowest cervical levels. ${ }^{1}$

The upper and lower parts of the cervical column are said to differ in their origin of development. Graber $^{2}$ suggested that the anatomy and position of the upper cervical vertebrae was closely linked with craniofacial development whereas the remaining cervical vertebrae developed in conjunction with the rest of the vertebral column. This was further demonstrated by Hellsing et $\mathrm{al}^{3}$ who reported that no correlation existed between cervical lordosis and thoracic kyphosis emphasizing on the close association of the cervical portion of the column with craniofacial development and not with the rest of the vertebral column.

Maxillo-mandibular relationship largely determines the facial profile of a patient. Hence, in orthodontics, studying skeletal vertical morphology as well as sagittal relations between the two jaws with reference to the cranial base forms an integral part of diagnosis and treatment planning. Cervical posture has previously been related to vertical craniofacial morphology. Dolicofacial faces are reported to have straight and forwardly inclined cervical columns while brachyfacial subjects have been found to have increased curvature of the spine. ${ }^{4,5}$ Similarly, correction of sagittal jaw relations by functional appliance therapy has shown to bring about changes in the inclination of the upper cervical column. This has been attributed to a change in the stomatognathic input provided by the craniofacial region. ${ }^{6}$ However a definitive link and association between sagittal jaw relations and cervical posture still remains unclear.

Since the cervical column lies in close proximity with the skeletal bases and has similar origins of development, determining correlation between the sagittal skeletal relations and cervical posture can enhance diagnosis as well as treatment prognosis. Hence, the objective of this study was to explore

Correspondence: Dr.Sanam Tauheed, Department of Orthodontics,Jinnah Medical and Dental College, Karachi,Sindh,Pakistan. Email: tauheedsanam@gmail.com .Phone: +92333-3121911.DOI: 10.3126/ jcmsn.v15i1.20509 Article received: 2018-07-06 Article accepted: 2018-09-21 
whether a significant difference in cervical posture exists in subjects with varying sagittal facial morphology i.e., different sagittal skeletal classes as well as investigating the level of correlation between the two.

\section{MATERIALS AND METHODS}

It was a cross-sectional study conducted at our orthodontic clinic. A total of 100 Pakistani subjects seeking orthodontic treatment were screened, out of which 63 subjects were selected on the basis of the inclusion criteria. Both males and females with fully erupted molars and premolars, with an age range of 11 to 22 years were included in the study. Individuals with any missing teeth, craniofacial anomalies, systemic disorders, temporomandibular joint disturbances, or subjects with upper airway obstruction were excluded. All selected subjects expressed their informed consent to participate in the study.

\section{Cephalometric Radiographs:}

Lateral cephalometric radiographs were taken in the natural head position with the teeth in occlusion. The natural head position of the patient was determined by positioning the patient in the cephalostat and adjusting the head using the mirror position. The mirror position was defined by instructing the patient to look in his own eyes, in a mirror set at eye-level opposite to the cephalostat. This ensured a standardized technique for recording the subjects' natural head and cervical column position. To ensure consistent magnification, all radiographs were made upon a standardized lateral radiograph $(18 \times 24 \mathrm{~cm}$ film, Kodak, Germany $)$ with a patient midplane- $X$ ray source distance of $146 \mathrm{~cm}$, patient midplane-film distance of $13.5 \mathrm{~cm}$ and enlargement factor of $1 \%$ by a single technician in our radiology department.

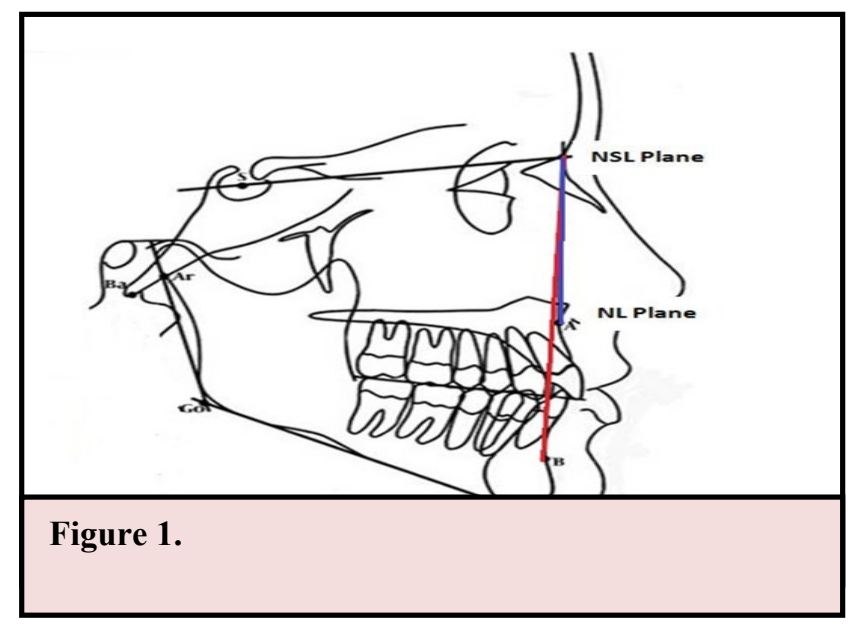

\section{Cephalometric Measurements:}

The lateral cephalometric radiographs were traced by the principle investigator and the maxillo- mandibular relationship was assessed using the angle $\mathrm{ANB}$, formed by joining points $\mathrm{A}, \mathrm{N}$ and $\mathrm{B}$ (Fig 1). This determined the sagittal jaw relationship with reference to the cranial base and has a normal value of $2 \pm 2^{\circ}$ (Class I). An angle larger than $4^{\circ}$ indicates a tendency to class II, and an angle smaller than $2^{\circ}$ indicates a Class III.

For assessing cervical curvature, reference points and lines were constructed as suggested by Solow and Tallgren7. A posterior tangent line to the odontoid process was constructed that passed both the most posterio-superior point (CV2SP) and posterio-inferior point (CV2IP) on the body of the second cervical vertebra. This was the Odontoid Process Tangent (OPT). The Cervical Vertebra Tangent (CVT) was a posterior tangent line to the odontoid process that passed the most posteriorsuperior point on the body of the second vertebra (CV2SP) and most posterior-inferior point on the body of the fourth cervical vertebra (CV4IP). The OPT and CVT planes cross the true horizontal (HOR plane- perpendicular to vertical plane of cephalometric film) making the cervicohorizontal angles - OPT/HOR and CVT/HOR respectively. These express the cervical inclination with reference to the true horizontal plane. The degree of curvature of the cervical column is determined by the angle OPT/CVT which is formed between the two tangents (Fig 2).

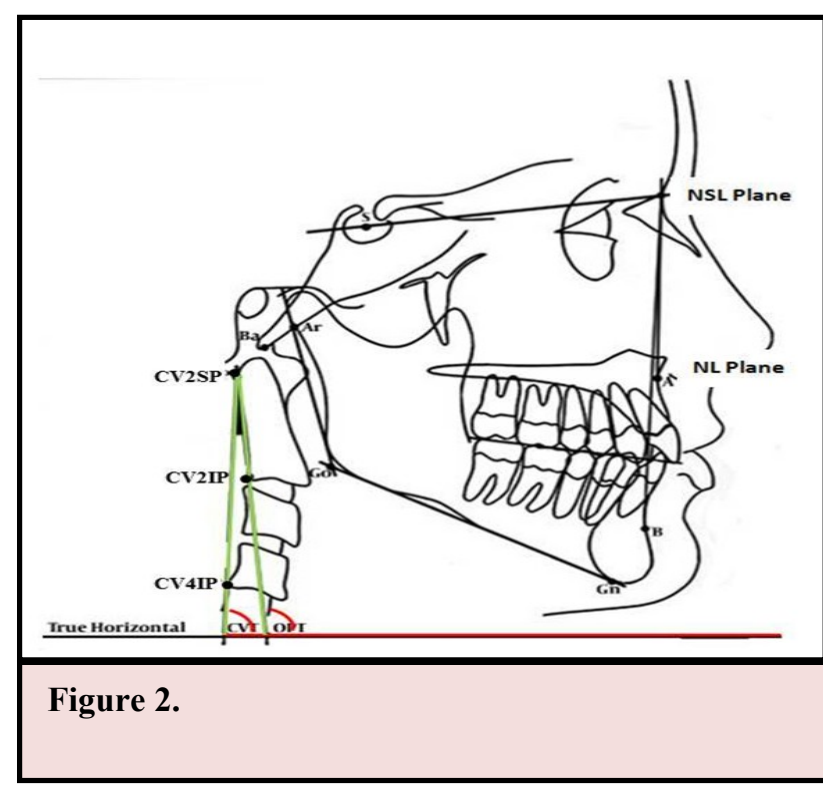

Measurement Error:

Intra-examiner reliability of the study variables was assessed by re-tracing and re-measuring 20 lateral cephalometric radiographs, selected at random from the study sample, by the principal investigator, two weeks after the initial data 
collection. Pearson's correlation was applied and a strong correlation $(r=0.8, p=0.02)$ was found

\section{Data Analysis:}

Data was analyzed using SPSS version 19.0 (Statistical Package for Social Sciences Chicago Inc). Descriptive statistics were computed for all variables. One way ANOVA was used to compare cervical inclination and curvature in different skeletal groups. Post Hoc Bonferroni test was further applied to test the difference between the three groups. Correlation of cervical posture with different skeletal malocclusions was tested using Pearson's correlation. Level of significance was set at $\mathrm{p} \leq 0.05$.

\section{RESULTS}

The total sample consisted of 63 patients out of which 25 were males and 38 were females. Mean age of male patients was 14 years and 8 months and of female patients was 15 years and 6 months. Based on the angle ANB, our data comprised of 22 subjects with Skeletal Class I, 21 with Skeletal Class II and 20 with skeletal Class III.

Table 1 shows a comparison of cervical posture in the different skeletal groups. Cervical curvature was found to differ in the three classes of malocclusion $(\mathrm{p}=0.025)$, namely skeletal Class I, II and III.

Table 1 shows a comparison of cervical posture in the different skeletal groups. Cervical curvature was found to differ in the three classes of malocclusion ( $p=0.025)$, namely skeletal Class I, II and III.

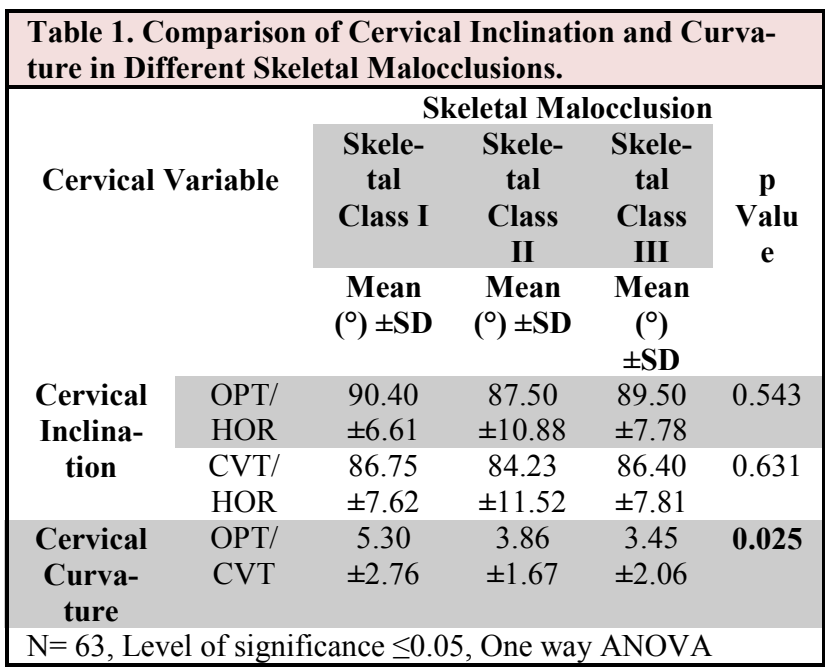

Table 2 displays an intergroup comparison in the different skeletal groups. Cervical curvature in subjects with Skeletal Class I base was found to be significantly different from that of Class III ( $p=$ 0.030). No statistically significant difference was seen between cervical curvature of skeletal Class I and II or between skeletal Class II and III. Difference in cervical inclination between the skeletal groups was also insignificant.

\begin{tabular}{|c|c|c|}
\hline \multirow{4}{*}{$\mathrm{p}$ Value } & $\begin{array}{l}\text { Malocclusion } \\
\text { Classes }\end{array}$ & $\begin{array}{c}\text { Cervical Curvature } \\
\text { (OPT/CVT) }\end{array}$ \\
\hline & I-II & 0.115 \\
\hline & I-III & $\mathbf{0 . 0 3 0}$ \\
\hline & II-III & 1.000 \\
\hline \multicolumn{3}{|c|}{$\mathrm{N}=63$, Level of significance $\leq 0.05$, Post Hoc Bonferroni } \\
\hline
\end{tabular}

Table 3 shows a correlation between cervical posture and skeletal malocclusions. Skeletal class presented a weak but significant correlation with only cervical curvature $(\mathrm{r}=0.30, \mathrm{p}=0.02)$. Cervical inclination was not found to be associated with skeletal Class.

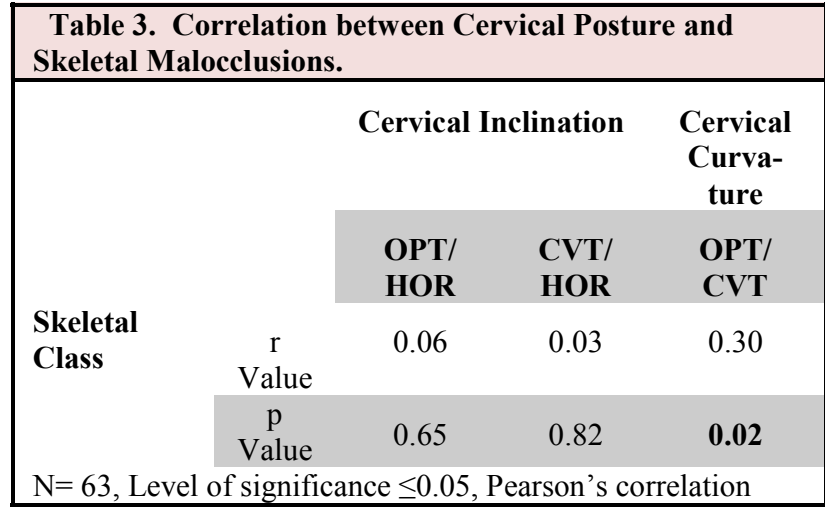

\section{DISCUSSION}

The present research was carried out to determine cervical posture in the different skeletal groups as well as to assess whether an association existed between cervical posture and sagittal skeletal relationships. Unlike previous studies ${ }^{8,9}$ which relied solely on visual assessment of cervical posture, the current study, utilized quantifiable cephalometric measurements to determine both cervical curvature and inclination. This increased the reliability and accuracy of the results.

The descriptive results of this study show that skeletal Class II subjects, on an average, had smaller cervicohorizontal angles, compared to dental Class I and III. This was indicative of a forward inclination of the cervical spine. On the other hand, dental Class III subjects had comparatively, larger cervicohorizontal angles which are representative of a backward positioned cervical spine. However, when the cephalometric measurements for cervical inclinations in different 
malocclusions were compared statistically, interestingly, no significant difference was found. This was in contrast to Alkofide et al's ${ }^{10}$ findings that showed a statistically significant difference in cervicohorizontal angles between Class I and II and between Class II and III.

In a descriptive comparison of the mean cervical curvature angles (OPT/CVT), it was seen that overall skeletal Class II and III subjects had lower mean values than Class I subjects. However, Class II subjects had slightly greater values than Class III. This was also noted by Mertensmeier and Diedrich $^{11}$ who showed that Class II subjects had greater lordosis of the spine compared to Class III patients. Data analysis showed that cervical curvature of skeletal Class III subjects was statistically different from Class I $(p=0.03)$. The degree of curvature for Class III subjects was significantly lesser than that of Class I indicating relatively straighter cervical columns. This was in agreement with some authors ${ }^{12-14}$ who affirm that Class III subjects tend to have head posteriorization and reduced cervical curvature compared to other malocclusions. D'Attilio et $\mathrm{al}^{15}$ also reported straighter cervical columns for skeletal Class III bases.

Skeletal Class showed a weak but significant correlation with cervical curvature OPT/CVT angle $(\mathrm{r}=0.30, \mathrm{p}=0.02)$. This was also observed by Hosseinzadeh et al, ${ }^{16}$ who reported a significant correlation between the angle ANB and their Modified Cervical Angle (OPT/CVT). This association emphasizes the possible relation between the posture of the cervical column and skeletal class and may prove to be of some aid to an orthodontist in order to confirm the diagnosis of a skeletal malocclusion at a glance just by looking at the patient's cervical vertebral curvature. Though, this was in contrast to Tecco et al's1 study who calculated cervical curvature of vertebrae 4 to 7 using the curve fitting method. They concluded that the amount of curvature and the ANB angle were not significantly correlated. As previously mentioned, maximum cervical lordosis occurs at the superior vertebrae mainly $\mathrm{C} 1$ and $\mathrm{C} 2$ and hence their insignificant result. Furthermore, the current study used quantifiable cephalometric measurements to assess cervical curvature in contrast to their curve fitting method. Our curvature angle OPT/CVT is more indicative of cervical curvature deviations and tracing of C6 vertebra is not required. It can be conveniently measured in the clinic and has a high level of reproducibility as seen by the insignificant level of measurement error seen here.

\section{CONCLUSIONS}

The present study concludes that skeletal sagittal jaw relations differ in their cervical postures, especially cervical curvature. Skeletal Class III subjects have significantly straighter cervical columns than skeletal Class I subjects. We also conclude that curvature of the cervical column has a correlation with sagittal jaw relations. Nevertheless, based on a cross-sectional study, it is not possible to reach a definitive conclusion that can indicate the cause and effect relation of cervical posture and skeletal sagittal jaw relations. Hence, it is recommended to follow these variables in a longitudinal study.

\section{REFERENCES}

1. Tecco S, Festa F. Cervical spine curvature and craniofacial morphology in an adult Caucasian group: a multiple regression analysis. Eur $\mathrm{J}$ Orthod 2007;29:204-9.

2. Graber TM 1958 Implementation of the roentgenographic cephalometric technique. Am J Orthod 1958;44:906 - 932.

3. Hellsing E, McWilliam J, Reigo T, Spangfort E. The relationship between craniofacial morphology, head posture and spinal curvature in 8,11 and 15-year-old children. Eur J Orthod 1987;9:254-64.

4. Solow B, Sandham A. Cranio-cervical posture: a factor in the development and function of the dentofacial structures. Eur J Orthod 2002;24:447-56

5. Solow B, Siersbaek-Nielsen S. Cervical and

craniocervical posture as predictors of raniofacial growth. Am J Orthod Dentofacial Orthop 1992;101:449-58.

6. Tecco S et al. 2005 Evaluation of cervical spine posture after functional therapy with FR2: a longitudinal study. Cranio. The Journal of Craniomandibular Practice 2005;23:53-66.

7. Solow B, Tallgren A. Head posture and craniofacial morphology. Am J Phys Anthropol 1976;44:417-35.

8. Solow B, Sonnesen L. Head posture and malocclusions. Eur J Orthod 1998;20:685-693.

9. Deda MR, Mello-Filho FV, Xavier SP, Trawitzki LV. Head posture in the presence of class II and class III dentofacial deformities. Rev. Cefac 2011.

10. AlKofide EA, AlNamankani E. The association between posture of the head and 
malocclusion in Saudi subjects. Cranio 2007;25:98-105.

11. Mertensmeier I, Diedrich P. The relationship between cervical spinal posture and bite anomalies. Fortschr Kieferorthop 1992;53:2632.

12. AlKofide EA, AlNamankani E. The association between posture of the head and malocclusion in Saudi subjects. Cranio 2007;25(2):98-105.

13. Martesmeier I, Diedrich P. Which correlations between cervical posture and malocclusions? Fortschritte der Kieferorthopadie. 1992;52:26-
32.

14. Biasotto-Gonzalez DAB. Abordagem interdisciplinar das disfunções temporomandibulares. São Paulo 2005: Manole

15. D’Attilio M, Caputi S, Epifania E, Festa F, Tecco S. Evaluation of cervical posture of children in skeletal Class I, II, and III. Cranio 2005;23:219-228.

16. Hosseinzadeh Nik T, Janbaz Aciyabar P. The Relationship Between Cervical Column Curvature and Sagittal Position of the Jaws: Using a New Method for Evaluating

Citation: Tauheed S, Shaikh A, Fida M. Cervical Posture and Skeletal Malocclusions - Is there a Link?JCMS Nepal. 2019;15(1):5-9 
